\title{
The value of liquid biopsy in the diagnosis and staging of hepatocellular carcinoma: a systematic review
}

\author{
Poh Tan ${ }^{1}$, Lisa Grundy ${ }^{1}$, Peter Makary ${ }^{1}$, Khem Hua Eng ${ }^{2}$, George Ramsay ${ }^{3}$, Mohamed Bekheit ${ }^{1,4}$ \\ ${ }^{1}$ Department of General Surgery, Aberdeen Royal Infirmary, Aberdeen, UK; ${ }^{2}$ Queens University Belfast, Belfast, UK; ${ }^{3}$ Rowette institute of Health \\ Sciences, Medical School, University of Aberdeen, Aberdeen, UK; ${ }^{4}$ Department of Surgery, El Kabbary Hospital, Alexandria, Egypt \\ Contributions: (I) Conception and design: M Bekheit, P Tan, P Makary; (II) Administrative support: None; (III) Provision of study materials or \\ patients: None; (IV) Collection and assembly of data: P Tan, P Makary, L Grundy; (V) Data analysis and interpretation: P Tan; (VI) Manuscript \\ writing: All authors; (VII) Final approval of manuscript: All authors. \\ Correspondence to: Mohamed Bekheit. Department of General Surgery, Aberdeen Royal Infirmary, Aberdeen, UK. \\ Email: dr_mohamedbekheit@hotmail.com.
}

\begin{abstract}
Background: Blood-borne tumour markers in the form of circulating tumour cells (CTCs) are of intense research interest in the diagnostic and prognostic work-up of hepatocellular carcinoma (HCC).

Methods: This is a meta-analysis. Using a PICO strategy, adults with HCC was the population, with the individual CTCs as the intervention and comparators. The primary outcome was the sensitivity and specificity of HCC detection with tumour specific single gene methylation alteration. Secondary outcomes were the comparison using specific assay methods and the effect of early $v$ s. late stages on CTC positivity. We included patients with HCC who had samples taken from peripheral blood and had sufficient data to assess the outcome data. ASSIA, Cochrane library, EMbase, Medline, PubMed and the knowledge network Scotland were systematically searched with appropriate Mesh terms employed. The quality assessment of diagnostic accuracy studies (QUADAS) was used to ensure quality of data. Statistical analysis was performed using the 'Rev Man' meta-analysis soft ward for Windows.

Results: The review included 36 studies, with a total of 5,853 patients. Here, we found that AFP has the highest overall diagnostic performance. The average Youden index amongst all CTC was 0.46 with a mode and median of 0.5 with highest of 0.87 and lowest of 0.01 .

Conclusions: The available literature provides weak evidence that there is potential in the use of CTC, however the lack of a standardised procedure in the study of CTC contribute to the lack of consensus of use. Future research should include large scaled, standardized studies for the diagnostic accuracy of CTCs.
\end{abstract}

Keywords: Hepatocellular carcinoma (HCC); tumour markers; circulating tumour cells (CTCs); liquid biopsy

Received: 12 September 2019; Accepted: 18 January 2020; Published: 25 October 2021.

doi: $10.21037 / \operatorname{tgh} .2020 .01 .11$

View this article at: http://dx.doi.org/10.21037/tgh.2020.01.11

\section{Introduction}

Hepatocellular carcinoma (HCC) is responsible for approximately $90 \%$ of primary liver cancers and is the second most common cause of cancer related deaths, worldwide (1). Although it is historically associated with viral infections, the incidence of HCC in western populations is expected to rise due to the increasing prevalence of noncommunicable diseases which are linked to this malignancy. Such diseases include obesity, diabetes, non-alcoholic fatty and alcoholic liver disease (2).

There have been recent changes to the available treatment options for HCC with concomitant improvements in outcome of patients with early disease. However, the overall prognosis of HCC remains generally poor, and is correlated with presenting stage. As such, early detection of the disease has been shown to be a significant clinical challenge. A diagnostic marker which has the 
capacity to detect early stage cancer is thus likely to alter the prognosis of $\mathrm{HCC}(3,4)$.

The current method by which HCC is diagnosed is primarily based on imaging methods. Whilst useful, they have a number of limitations. Ultrasound is operator dependent and the sensitivity may be diminished due to body habitus. Although CT scan has demonstrated marked increase in sensitivity and specificity in comparison to US scan, studies have indicated an indirect correlation to tumour size which limits its ability in diagnosis of early cancers (5). MRI is more sensitive (6), however, these scans are expensive, time consuming and are resource constrained.

A number of candidate markers are available which may represent breakthroughs in future HCC diagnosis and management. Blood borne tumour markers in the form of circulating tumour cells (CTCs) (7) and cell free nucleic acids (cfDNA) (8) are topics of intense research. Each may allow for strategies to detect cancers in early stages, measure treatment progress, and offer prognosis post treatment. CTCs have been identified as playing a large role in metastasis and recurrence via their nature of shedding of the primary tumour into blood, lymph and bone marrow which allows circulation to other parts of the body (8). However, as a tumour marker, detection of CTCs remains limited at best due to the lack of volume sensitivity in early stage HC, and a wide specific detection range complicated by common hepatic diseases associated with HCC (7).

CTCs are suggested to hold exciting potentials and many studies have been conducted to discover new possibilities. However, the sensitivity and specificity remain unclear. To ensure high fidelity in this novel topic, this meta-analysis aims to determine the diagnostic accuracy of CTC in the diagnosis of HCC.

\section{Methods}

This was a meta-analysis carried out according to the PRIMA guidelines (9).

\section{Eligibility criteria and outcomes}

Studies eligible for the analysis were defined using the Population, Intervention, Comparison and Outcome strategy (PICO) (10). The study population was adults who were diagnosed with HCC via current gold standards methods of detection. Type of intervention was the use of CTCs in the detection of HCC. Comparator was individual CTC performance in the study. The primary outcome was the overall sensitivity and specificity of HCC detection with tumour specific single gene methylation alteration. Secondary outcomes were the comparison using specific assay methods, early $v s$. late HCC stage and specific TNM staging and Youden index.

\section{Inclusion criteria}

Studies were included if they matched all of the following: (I) all patients were diagnosed with HCC; (II) samples taken were from peripheral blood; (III) sufficient data was available to assess sensitivity and specificity of the CTC or data were available to calculate from primary data.

Studies were excluded if insufficient data for describing or calculating sensitivity and specificity values; sample evaluation was not related to HCC; full papers were unavailable or the publication type was either letters to the editors, reviews, technical reports, case reports. Articles written in languages other than English and non-human studies were also excluded from the study.

\section{Literature search}

ASSIA, Cochrane library, EMbase, Medline, PubMed and the knowledge network Scotland were systematically searched. The search criteria included a combination of Mesh and string terms, for the following searches in each database: (I) ("liquid biopsy" OR "liquid biopsies") AND "Liver Neoplasms"(Mesh). (II) "Liver Neoplasms"(Mesh) AND ("cfdna" OR "cell-free DNA"); (III) "Liver Neoplasms"(Mesh) AND Neoplastic cells, Circulating"(Mesh). "Liver Neoplasms"(Mesh) was substituted with string terms "liver cancer", "liver neoplasm", "liver tumor", "hcc", and "primary liver tumor" in cases where the Mesh term could not be utilised.

There was no limit on the date of publication and the search was updated till February 2019.

\section{Study selection and data extraction}

PT, PM, LG conducted their database search independently.

Titles and abstracts were analysed and the studies were uploaded on to Rayyan QRCI (11) to be reviewed manually by the authors. The abstracts were screened on Rayyan QRCI and any disagreements on data extraction and quality assessment of the included studies were resolved through discussion and checked by MB. The final included studies were uploaded to Mendeley ${ }^{\circledR}$, Elsevier, London, UK (12). A 
PRISMA 2009 Flow Diagram

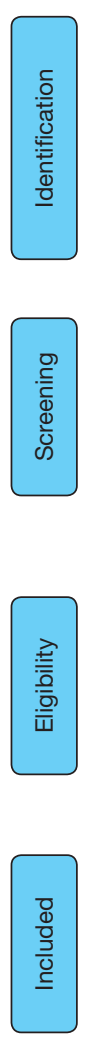

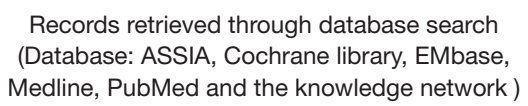
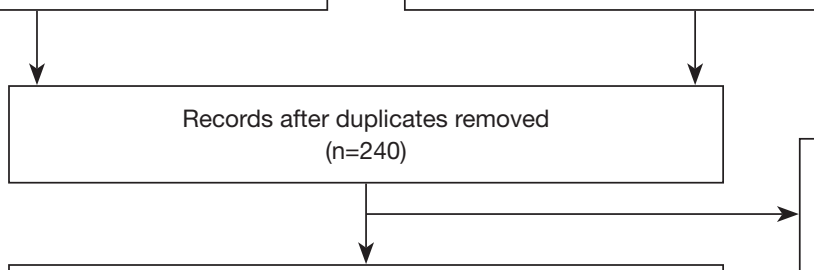

Excluded $(n=159)$

* Non relevant $(n=150)$

* Letter or reply $(\mathrm{N}=9)$

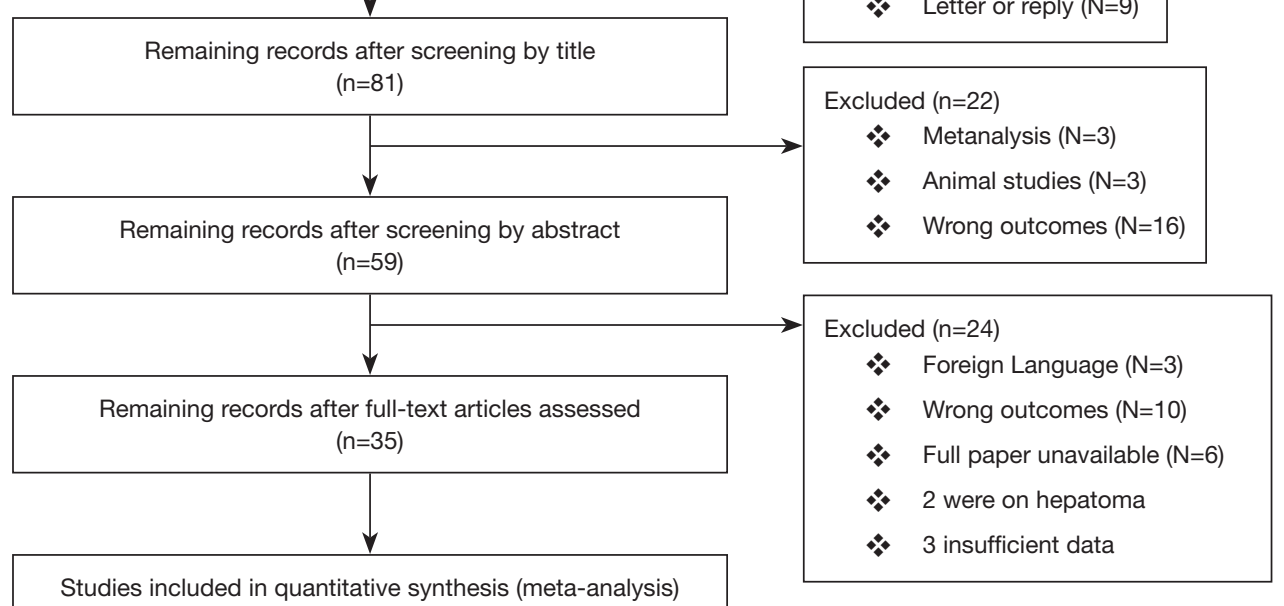

Studies included in quantitative synthesis (meta-analysis) $(n=35)$

Figure 1 Flow chart for inclusion and exclusion of studies in the meta-analysis.

flow chart for the inclusions and exclusions of this study is show in Figure 1.

Full text articles were matched in Mendeley and further examine using the criteria stated below.

The data extracted from the articles were publication year, participant demographics, experimental method, assay indicators, cut off values, CTC positivity, cancer stages and sensitivity and specificity scorings.

The data were then categorized and analysed based four distinct subgroup. They were (I) CTCs detected in serum; (II) CTC detection methodology; (III) low stage cancers; (IV) CTC detection rates and (V) group comparing the positivity rates of low and high stage cancers.

\section{Quality assessment}

The quality score of the studies were judged based on the Quality assessment of diagnostic accuracy studies (QUADAS) (13). The quality assessment is stratified into 14 item phrased questions each with yes, no or unclear. The questions covered 4 domains patient selection, index test, reference standard, and flow and timing. Each domain is assessed in terms of risk of bias, and the first 3 domains are also assessed in terms of concerns regarding applicability. The maximum score is 14, a score of 7 or greater indicated a high-quality study, whilst less than 7 were of low quality (Table 1).

\section{Statistical analysis}

Statistical analysis was performed using the 'Rev Man' metaanalysis soft ward for Windows. This software managed all the data and generated all the forest plots and the MosesLittenberg SROC curve.

The study performed the diagnostic accuracy test review by calculating the sensitivity and specificity of each test. The data was tabulated and produced a scatter plot (Figures 2,3). Using the Moses-Littenberg SROC curve on rev man a summary roc curve was produced for each data set which gives an indication for descriptive purposes (Figure 4). 
Table 1 Summary of studies included in the meta-analysis

\begin{tabular}{|c|c|c|c|c|c|c|c|c|c|c|c|c|c|c|c|}
\hline First author & Year & $\begin{array}{c}\text { QUADAS } \\
\text { score }\end{array}$ & Patients & $M / F$ & Sample & Assay method & Assay indicators & Cutoff & TP & $\mathrm{FP}$ & $\mathrm{TN}$ & $\mathrm{FN}$ & Sensitivity & Specificity & $\begin{array}{c}\text { Youden } \\
\text { index }\end{array}$ \\
\hline Aselmann et al. (14) & 2001 & 6 & 66 & NA & Blood & RT-PCR & Methylation (AFP) & $14 \mathrm{ng} / \mathrm{mL}$ & 6 & 5 & 39 & 16 & 13 & 89 & 0.02 \\
\hline \multirow[t]{4}{*}{ Bahnassy et al. (15) } & 2014 & 6 & 183 & $121 / 12$ & Blood & RT-PCR & Methylation (AFP) & $7.5 \mathrm{ng} / \mathrm{mL}$ & 115 & 5 & 57 & 6 & 96 & 91 & 0.87 \\
\hline & 2014 & 6 & 183 & $121 / 13$ & Blood & RT-PCR & Methylation (CD133) & $73 \mathrm{ng} / \mathrm{mL}$ & 48 & 72 & 4 & 59 & 40 & 6 & 0.54 \\
\hline & 2014 & 6 & 183 & $121 / 14$ & Blood & RT-PCR & Methylation (CK19) & $49.8 \mathrm{ng} / \mathrm{mL}$ & 105 & 15 & 51 & 12 & 87 & 81 & 0.72 \\
\hline & 2014 & 6 & 183 & $121 / 15$ & Blood & RT-PCR & Methylation (CD90) & $400 \mathrm{ng} / \mathrm{mL}$ & 99 & 21 & 56 & 7 & 83 & 90 & 0.68 \\
\hline \multirow[t]{5}{*}{ Chang et al. (16) } & 2008 & 6 & 37 & NA & Plasma & MS-PCR & Methylation (APC) & N/A & 14 & 12 & 6 & 5 & 62 & 88 & 0.49 \\
\hline & 2008 & 6 & 37 & NA & Plasma & MS-PCR & Methylation (P16) & N/A & 15 & 9 & 4 & 9 & 79 & 50 & 0.20 \\
\hline & 2008 & 6 & 37 & NA & Plasma & MS-PCR & Methylation (GSTP1) & $\mathrm{N} / \mathrm{A}$ & 13 & 6 & 6 & 12 & 68 & 67 & 0.35 \\
\hline & 2008 & 6 & 37 & NA & Plasma & MS-PCR & Methylation (RASSF1A) & N/A & 12 & 4 & 7 & 14 & 63 & 78 & 0.29 \\
\hline & 2008 & 6 & 37 & NA & Plasma & MS-PCR & Methylation (Ecadherin) & $\mathrm{N} / \mathrm{A}$ & 18 & 14 & 1 & 4 & 98 & 22 & 0.41 \\
\hline Chen et al. (17) & 2012 & 9 & 210 & $144 / 66$ & Blood & qPCR & Methylation (cfDNA) & $213.8 \mathrm{ng} / \mathrm{mL}$ & 69 & 11 & 103 & 27 & 86 & 79 & 0.54 \\
\hline \multirow[t]{6}{*}{ Chen et al. (18) } & 2013 & 8 & 84 & $68 / 17$ & Serum & Chemiluminescense & Methylation (cfDNA) & N/A & 22 & 17 & 37 & 8 & 56 & 96 & 0.45 \\
\hline & 2013 & 8 & 84 & $68 / 18$ & Serum & Chemiluminescense & Methylation (AFP) & N/A & 21 & 18 & 36 & 9 & 54 & 91 & 0.42 \\
\hline & 2013 & 8 & 84 & $68 / 19$ & Serum & Chemiluminescense & Methylation (AFU) & N/A & 26 & 13 & 29 & 16 & 67 & 76 & 0.52 \\
\hline & 2013 & 8 & 84 & $68 / 20$ & Serum & Chemiluminescense & Methylation (cfDNA + AFP) & N/A & 28 & 11 & 34 & 11 & 72 & 87 & 0.65 \\
\hline & 2013 & 8 & 84 & $68 / 21$ & Serum & Chemiluminescense & Methylation (CFDNA + AFU) & $\mathrm{N} / \mathrm{A}$ & 34 & 5 & 28 & 17 & 87 & 71 & 0.59 \\
\hline & 2013 & 8 & 84 & $68 / 22$ & Serum & Chemiluminescense & Methylation (CFDAN + AFP + AFU) & N/A & 35 & 4 & 25 & 20 & 90 & 64 & 0.58 \\
\hline Chu et al. (19) & 2004 & 7 & 69 & $45 / 24$ & Serum & MS-PCR & Methylation (P16) & N/A & 22 & 4 & 19 & 24 & 48 & 83 & 0.30 \\
\hline \multirow[t]{3}{*}{ Fu et al. (20) } & 2017 & 7 & 1647 & NA & Blood & ELISA & Methylation (Hsp90a) & $62.44 \mathrm{ng} / \mathrm{mL}$ & 838 & 66 & 4 & 677 & 93 & 90 & 0.67 \\
\hline & 2017 & 7 & 1647 & NA & Blood & ELISA & Methylation (AFP20 & $6.98 \mathrm{ng} / \mathrm{mL}$ & 678 & 226 & 686 & 517 & 75 & 92 & 0.49 \\
\hline & 2017 & 7 & 1647 & NA & Blood & ELISA & Methylation (HSP90a+AFP) & N/A & 847 & 57 & 701 & 686 & 94 & 94 & 0.39 \\
\hline \multirow[t]{6}{*}{ Han et al. (21) } & 2014 & 11 & 293 & $221 / 72$ & Blood & MS-PCR & Methylation (AFP) & $20 \mathrm{ng} / \mathrm{mL}$ & 93 & 46 & 87 & 67 & 58 & 48 & 0.20 \\
\hline & 2014 & 11 & 293 & $221 / 73$ & Blood & MS-PCR & Methylation (AFP) & $200 \mathrm{ng} / \mathrm{mL}$ & 49 & 7 & 126 & 111 & 31 & 92 & 0.06 \\
\hline & 2014 & 11 & 293 & $221 / 74$ & Blood & MS-PCR & Methylation (AFP) & $400 \mathrm{ng} / \mathrm{mL}$ & 39 & 1 & 132 & 121 & 24 & 99 & 0.23 \\
\hline & 2014 & 11 & 293 & $221 / 75$ & Blood & MS-PCR & Methylation (TRG5 + AFP) & $20 \mathrm{ng} / \mathrm{mL}$ & 130 & 54 & 79 & 30 & 81 & 39 & 0.23 \\
\hline & 2014 & 11 & 293 & $221 / 76$ & Blood & MS-PCR & Methylation (TRG5 + AFP) & $200 \mathrm{ng} / \mathrm{mL}$ & 109 & 19 & 114 & 51 & 68 & 78 & 0.47 \\
\hline & 2014 & 11 & 293 & $221 / 77$ & Blood & MS-PCR & Methylation (TRG5 + AFP) & $400 \mathrm{ng} / \mathrm{mL}$ & 104 & 13 & 120 & 56 & 65 & 85 & 0.50 \\
\hline Hosny et al. (22) & 2008 & 8 & 255 & NA & Tissue & PCR & Methylation (249ser P53) & NA & 1 & 168 & 11 & 75 & 1 & 94 & 0.28 \\
\hline Huang et al. (23) & 2003 & 8 & 75 & $67 / 18$ & Blood samples & RT PCR & Methylation (249ser P53) & $254 \mathrm{ng} / \mathrm{mL}$ & 10 & 6 & 44 & 15 & 40 & 88 & 0.80 \\
\hline \multirow[t]{3}{*}{ Huang et al. (24) } & 2015 & 5 & 109 & NA & Serum & Pyrosequencing & Methylation (P16) & $5 \%$ & 49 & 8 & 35 & 17 & 65 & 87 & 0.38 \\
\hline & 2015 & 5 & 109 & NA & Serum & Pyrosequencing & Methylation (P16) & $7 \%$ & 33 & 2 & 41 & 33 & 39 & 97 & 0.53 \\
\hline & 2015 & 5 & 109 & NA & Serum & Pyrosequencing & Methylation (P16) & $10 \%$ & 18 & 1 & 42 & 48 & 20 & 99 & 0.36 \\
\hline \multirow[t]{2}{*}{ Huang et al. (25) } & 2012 & 8 & 150 & $61 / 11$ & Plasma & $R Q-P C R$ & Quantitative analysis of CfDNA & $18 \mathrm{ng} / \mathrm{mL}$ & 65 & 7 & 71 & 7 & 90 & 90 & 0.19 \\
\hline & 2012 & 8 & 150 & $61 / 12$ & Plasma & RQ-PCR & Quantitative analysis of CfDNA & $143 \mathrm{ng} / \mathrm{mL}$ & 43 & 29 & 49 & 29 & 60 & 78 & 0.07 \\
\hline Igetei et al. (26) & 2008 & 8 & 162 & NA & Plasma & Nested-PCR & Methylation (249ser P53) & $\mathrm{N} / \mathrm{A}$ & 6 & 0 & 77 & 79 & 7 & 100 & 0.43 \\
\hline lizuka et al. (27) & 2006 & 9 & 82 & $65 / 35$ & Serum & Real-time PCR & Quantitative analysis CfDNA & $73 \mathrm{ng}$ & 36 & 16 & 14 & 16 & 69 & 93 & 0.63 \\
\hline \multirow[t]{4}{*}{ lizuka et al. (28) } & 2010 & 11 & 258 & $123 / 135$ & Serum & MS-PCR & Methylation (SRD5A2) & NA & 4 & 144 & 2 & 108 & 4 & 99 & 0.50 \\
\hline & 2010 & 11 & 258 & $123 / 135$ & Serum & MS-PCR & Methylation (SPINT2) & NA & 18 & 146 & 0 & 94 & 16 & 100 & 0.01 \\
\hline & 2010 & 11 & 258 & $123 / 135$ & Serum & MS-PCR & Methylation (AFP) & $20 \mathrm{ng} / \mathrm{mL}$ & 68 & 105 & 41 & 44 & 86 & 67 & 0.33 \\
\hline & 2010 & 11 & 258 & $123 / 135$ & Serum & MS-PCR & Methylation (PIVKA-2) & $40 \mathrm{mAU} / \mathrm{ml}$ & 59 & 138 & 8 & 53 & 89 & 70 & 0.50 \\
\hline Jeng et al. (29) & 2004 & 7 & 111 & $57 / 54$ & Blood & RT-PCR & Methylation (AFP mRNA) & $1,000 \mathrm{ng} / \mathrm{mL}$ & 61 & 20 & 28 & 2 & 75 & 93 & 0.50 \\
\hline
\end{tabular}

Table 1 (continued) 


\begin{tabular}{|c|c|c|c|c|c|c|c|c|c|c|c|c|c|c|c|}
\hline First author & Year & $\begin{array}{c}\text { QUADAS } \\
\text { score }\end{array}$ & Patients & $\mathrm{M} / \mathrm{F}$ & Sample & Assay method & Assay indicators & Cutoff & TP & FP & TN & $\mathrm{FN}$ & Sensitivity & Specificity & $\begin{array}{c}\text { Youden } \\
\text { index }\end{array}$ \\
\hline Ji et al. (30) & 2014 & 9 & 289 & $100 / 21$ & Serum & MS-PCR & Methylation (MT1G) & NA & 85 & 36 & 6 & 62 & 70 & 91 & 0.50 \\
\hline Jiang et al. (31) & 2015 & 8 & 205 & NA & Serum/plasma & RT-PCR & Methylation (HBB) & & 76 & 14 & 32 & 83 & 80 & 94 & 0.50 \\
\hline \multirow{3}{*}{$\begin{array}{l}\text { Julich-Haertel et al. } \\
\text { (32) }\end{array}$} & 2017 & 8 & 517 & $310 / 145$ & Serum/plasma & Fluorescence & Methylation (taMPs) & 9.3 & 63 & 23 & 355 & 76 & 73 & 82 & 0.50 \\
\hline & 2017 & 8 & 517 & $310 / 146$ & Serum/plasma & Fluorescence & Methylation (taMPs) & 4.305 & 60 & 26 & 178 & 253 & 70 & 41 & 0.50 \\
\hline & 2017 & 8 & 517 & $310 / 147$ & Serum/plasma & Fluorescence & Methylation (taMPs) & 4.125 & 70 & 16 & 202 & 229 & 81 & 47 & 0.50 \\
\hline Kirk et al. (33) & 2000 & 8 & 119 & $97 / 22$ & Blood & PCR & Methylation (249ser P53) & NA & 19 & 5 & 61 & 34 & 36 & 92 & 0.50 \\
\hline Kuo et al. (34) & 2014 & 8 & 83 & NA & Plasma & MS-PCR & Methylation (HOXA9) & $\mathrm{Ml}>0.88$ & 23 & 14 & 42 & 7 & 73.3 & 97.1 & 0.50 \\
\hline Mohamed et al. (35) & 2012 & 8 & 100 & $70 / 30$ & Serum & Real-time PCR & Methylation (RASSF1A) & $640 \mathrm{nmol}$ & 36 & 25 & 35 & 4 & 90 & 55 & 0.50 \\
\hline Müller et al. (36) & 1997 & 8 & 91 & 12-Jul & Blood & PCR & Methylation (Albumin mRNA) & NA & 12 & 48 & 24 & 7 & 33 & 33 & 0.50 \\
\hline Piciocchi et al. (37) & 2013 & 10 & 142 & $99 / 43$ & Plasma & Real-time PCR & Quantitative analysis CfDNA & $1 \mathrm{ng}$ & 40 & 31 & 45 & 26 & 91 & 43 & 0.50 \\
\hline Ren et al. (38) & 2006 & 8 & 119 & NA & Plasma & Transilluminator & Quantitative analysis CfDNA & $36.6 \mathrm{ng}$ & 41 & 38 & 9 & 31 & 52 & 95 & 0.50 \\
\hline Schulze et al. (39) & 2013 & 7 & 78 & $60 / 18$ & Blood & CellSearch & Methylation (EpCAM) & & 18 & 1 & 18 & 41 & 56 & 92 & 0.50 \\
\hline Sun et al. (40) & 2013 & 10 & 93 & $36 / 7^{y}$ & Serum & MS-PCR & Methylation (TFPI2) & NA & 20 & 9 & 41 & 23 & 47 & 82 & 0.50 \\
\hline Tan et al. (41) & 2007 & 8 & 18 & NA & Serum & MS-PCR & Methylation (RUNX3) & NA & 7 & 1 & 0 & 10 & 88 & 100 & 0.50 \\
\hline Witzigmann et al. (42) & 2002 & 5 & 201 & $118 / 92$ & Blood & RT-PCR & Methylation (AFP mRNA) & & 24 & 3 & 113 & 61 & 73 & 53 & 0.50 \\
\hline Wong et al. (43) & 2003 & 6 & 100 & NA & Serum/plasma & MS-PCR & Methylation (P16) & NA & 24 & 21 & 0 & 55 & 53 & 100 & 0.50 \\
\hline Briefs et al. (44) & 1998 & 7 & 95 & NA & Serum/plasma & MS-PCR & Methylation (P16) & NA & 14 & 31 & 0 & 50 & 31 & 100 & 0.50 \\
\hline Yang et al. (45) & 2005 & 6 & 130 & NA & Blood & RT-PCR & Methylation (AFPmRNA) & & 35 & 3 & 62 & 30 & 54 & 95 & 0.50 \\
\hline Okajima (46) & 2017 & 8 & 110 & $79 / 31$ & Plasma & RQ-PCR & Quantitative analysis CfDNA & $1.87 \times 10$ & 38 & 22 & 5 & 45 & 64 & 90 & 0.50 \\
\hline Yeo et al. (47) & 2005 & 10 & 50 & $33 / 7^{y}$ & Plasma & MS-PCR & Methylation (RASSF1A) & NA & 17 & 23 & 0 & 10 & 43 & 100 & 0.50 \\
\hline Zhang et al. (48) & 2013 & 8 & 58 & $30 / 28$ & Serum & Chip/Pyrosequencing & Methylation (DBX2) & NA & 28 & 3 & 3 & 24 & 88.9 & 87 & 0.50 \\
\hline \multirow[t]{4}{*}{ Zhang et al. (49) } & 2007 & 12 & 100 & $78 / 22$ & Serum & MS-PCR & Methylation (P16) & NA & 22 & 28 & 2 & 48 & 44 & 96 & 0.50 \\
\hline & 2007 & 12 & 58 & $78 / 23$ & Serum & Chip/Pyrosequencing & Methylation (THY1) & NA & 26 & 5 & 5 & 22 & 85 & 81 & 0.50 \\
\hline & 2007 & 12 & 100 & $78 / 24$ & Serum & MS-PCR & Methylation (P15) & NA & 11 & 39 & 0 & 50 & 22 & 100 & 0.50 \\
\hline & 2007 & 12 & 100 & $78 / 25$ & Serum & MS-PCR & Methylation (RASSF1A) & NA & 35 & 15 & 3 & 47 & 70 & 94 & 0.02 \\
\hline
\end{tabular}

QUADAS, quality assessment of diagnostic accuracy studies; M/F, male/female; TP, true positive; FP, false positive; TN, true negative; FN, false negative.

Studies which compared CTC positivity in high and low stages of cancer were plot on a graph and compared (Figure 5).

\section{Results}

In this review, 240 studies were initially identified in the literature search. After analysing titles and abstracts, there were 181 studies excluded and 59 potential studies which were further reviewed. Of the 59 studies, 24 were excluded as they did not meet the inclusion criteria (Figure 1). Finally, 35 studies were compliant with the inclusion criteria and were eligible for the meta-analysis (Table 1).

There were a total of 5,945 patients, of whom 2,344 were male. All patients involve were diagnosed with HCC. Samples were taken from patient blood in 13 groups, serum in 12, plasma 7 in and 4 had samples taken from both serum and plasma.

All studies were published from 1994 onward. The flow chart of inclusion and exclusion studies is presented in the figure below. The average quadas score was 7.8 with lowest at 5 and highest 12 .

From the forty one [41] studies, twenty four [24] evaluated the use of a tumour specific single gene methylation. Eleven trials assessed patients of the same cancer stage comparing the positivity rates of each CTC demonstrated in graphical form (Figure 4).

In addition, the method of CTC detection was analysed. There were 5 methods of analysis including chemiluminescence [variation of the standard enzyme immunoassay (EIA), which is a biochemical technique used in immunology], ELISA, MS-PCR and Real-time polymerase chain reaction (RT PCR). These methods of analysis had 


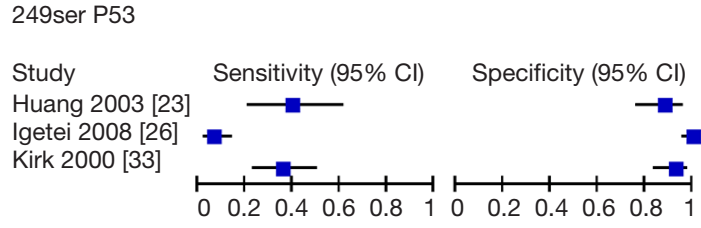

cfDNA

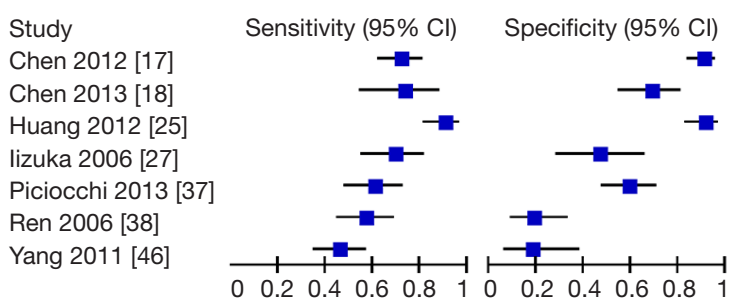

RASSF1A

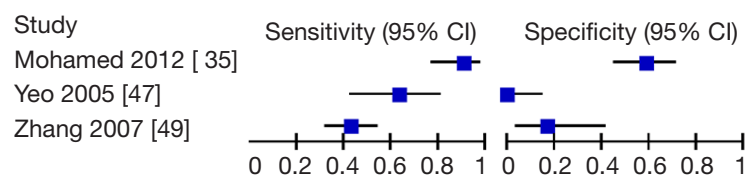

AFP

\section{Study}

Aselmann 2001 [14]

Bahnassy 2014 [15]

Chen 2013 [18]

Fu Y 2017 [20]

Han 2014 [21]

izuk 2011 [28]

Jeng 2004 [29]

Witzigmann 2002 [42]

Yang 2005 [45]

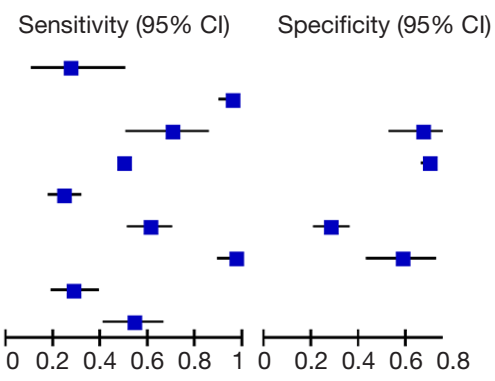

P16

Study

Chang 2008 [16]

Chu 2004 [19]

Huang 2014 [24]

Huang 2014 [24]

Huang 2014 [24]

Wong 2000 [43]

Wong 2003 [44]

Zhang 2007 [49]
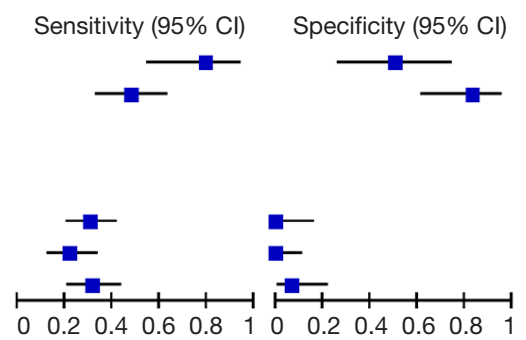

Figure 2 Forest plots of estimates of sensitivity and specificity for the different markers $(14-21,23-29,33,35)$ constituting liquid biopsy $(37,38,42-47,49)$.

Various assay method for AFP

$\begin{array}{ll}\text { Study } & \text { Assay method } \\ \text { Aselmann } 2001 \text { et al (AFP) [14] } & \text { RT PCR } \\ \text { Bahnassy et al 2014 (AFP) [15] } & \text { RT PCR } \\ \text { Chen et al 2013 (AFP) [18] } & \text { Chemiluminescence } \\ \text { Fu } \mathrm{Y} 2017 \text { (AFP20) [20] } & \text { ELISA } \\ \text { Han et al 2014 (AFP) [21] } & \text { MS PCR } \\ \text { lizuka et al 2011 (AFP) [28] } & \text { MS PCR } \\ \text { Jeng et al 2004 (AFP mRNA) [29] } & \text { RT PCR } \\ \text { Witzigmann et al 2002 (AFP mRNA) [42] } & \text { RT PCR } \\ \text { Yang et al 2005 (AFPmRNA) [47] } & \text { RT PCR }\end{array}$

Figure 3 Forest plots of estimates of sensitivity and specificity for the different assay methodology $(14,15,18,20,21,28,29,42,47)$.

6 studies, 3 studies, 15 studies and 15 studies respectively.

\section{Sensitivity and specificity of different circulating tumor cells}

Four studies observed the 249 ser p53 CTC, which all have high specificity $>0.8$ but moderate to low sensitivity which is indicated on part of the roc curve. Alpha feto protein (AFP) has 8 studies all of which have moderate to high sensitivity and a high specificity $>0.6$. Circulating free DNA
(CfDNA) has 4 studies with high the highest sensitivity and specificity, most of the studies above 0.6. cFDNA show promising result in comparison to the other liquid biopsy. P16 and Ras association domain-containing protein 1 (RASSF1A) had the lowest sensitivity and specificity. The SROC curves were incomplete denoting uncertainty. The observed data is presented graphically on the forest plot (Figure 3) and SROC curve (Figure 5). Further analysis demonstrated that CD133, CK19, CD90 could be used to provide discriminatory values between the early and late 
AFP

Study

Han 2014 [21]

Han 2014 [21]

Han 2014 [21]

TRG5 + AFP

Study

Han 2014 [21]

Han 2014 [21]

Han 2014 [21]
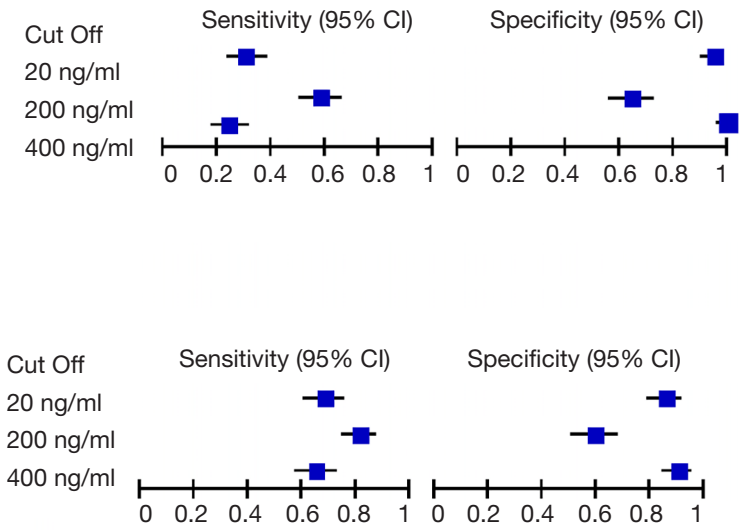

P16

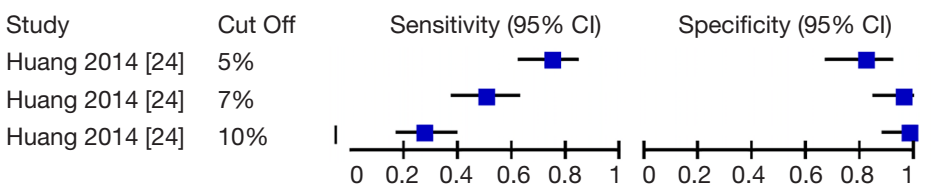

Figure 4 Forest plots of comparing the specificity and sensitivity of different circulating tumour cells at different cut off $(21,24)$.
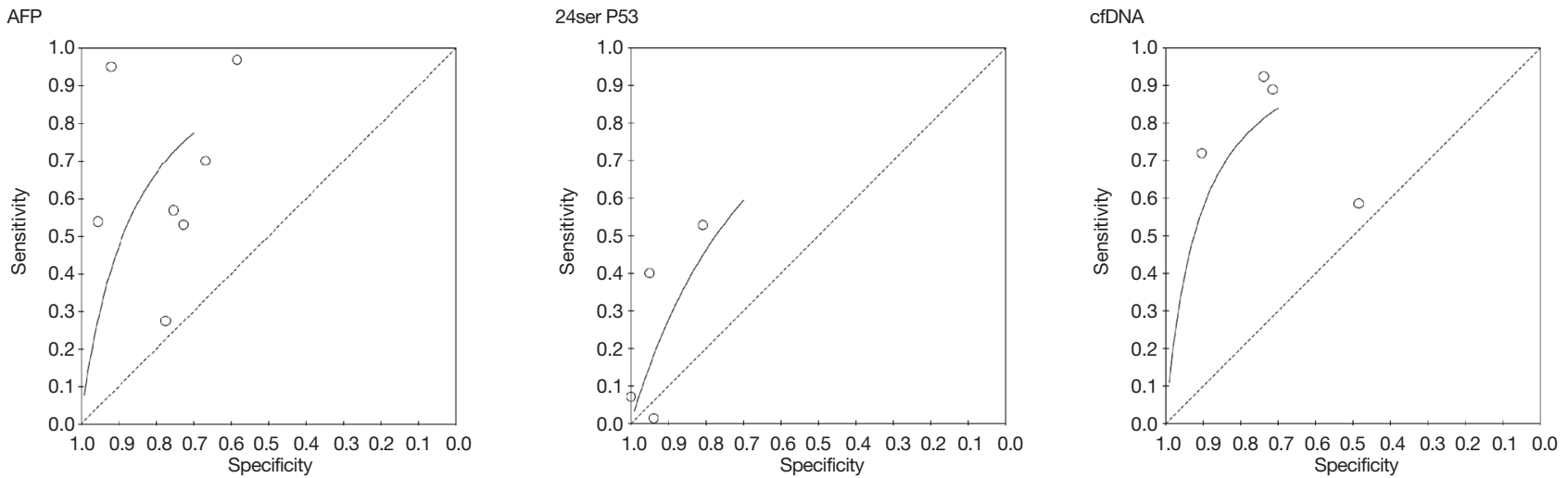

$\mathrm{P} 16$
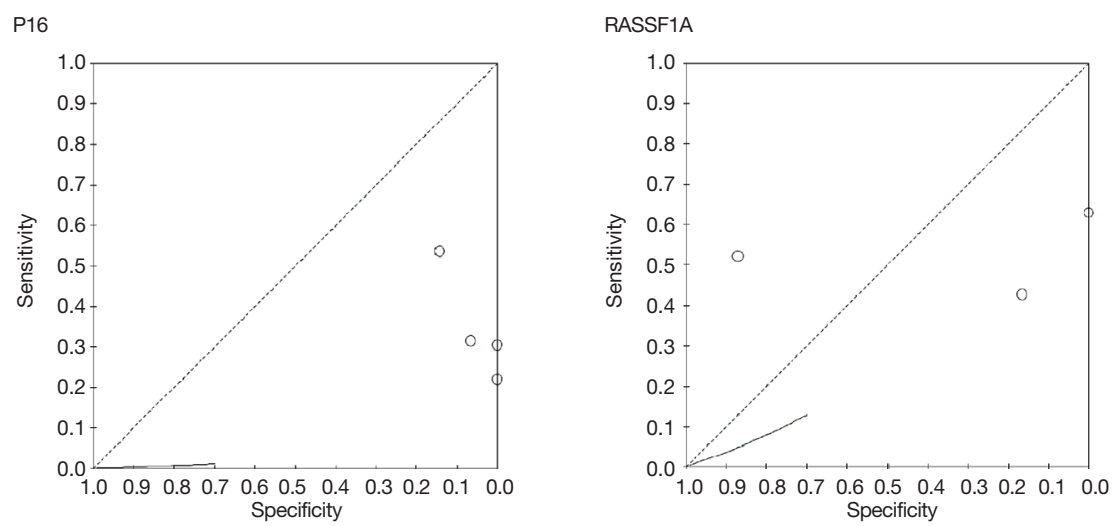

Figure 5 SROC curve for different circulating tumour cells. 


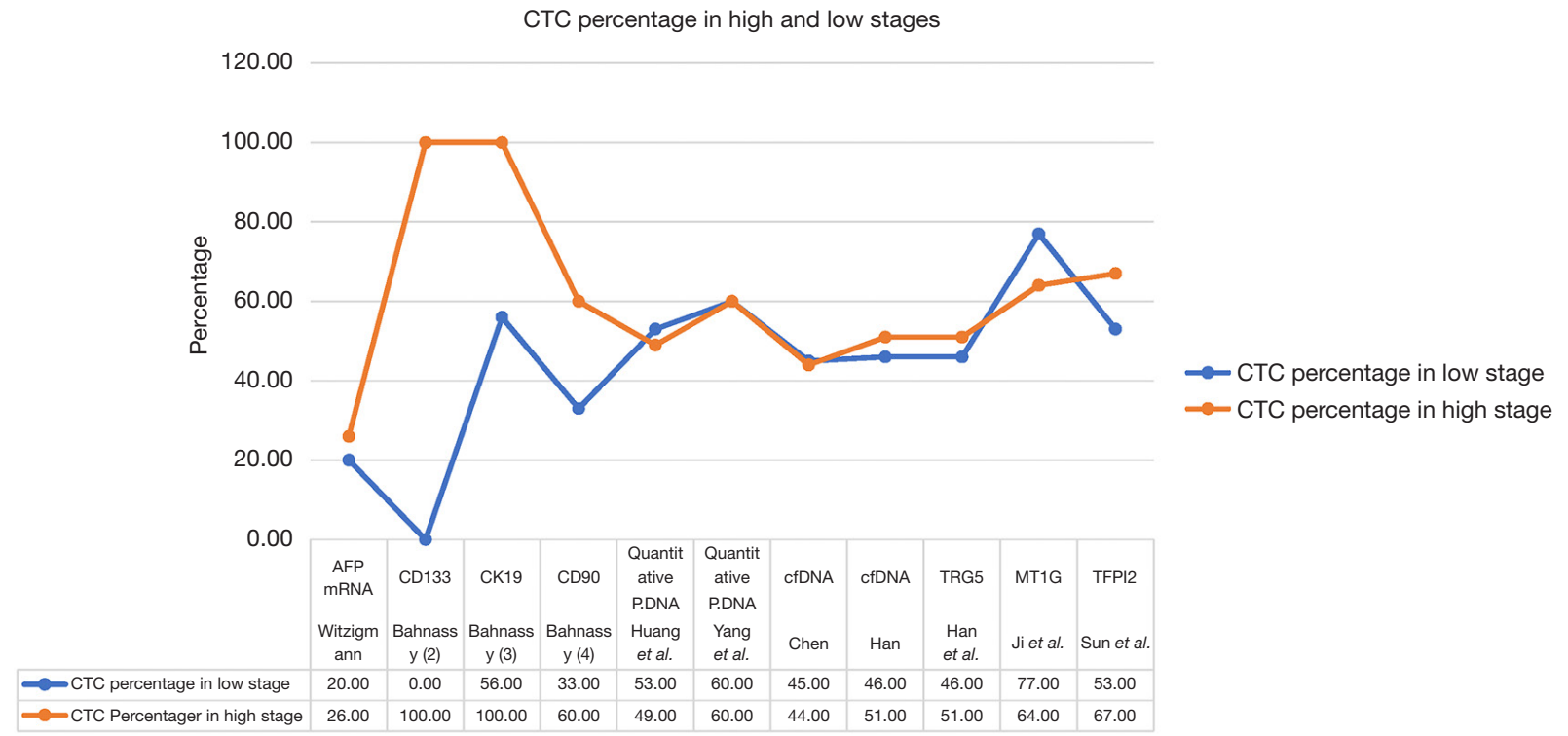

Figure 6 Positivity of CTC in late stages $(3 \& 4)$ and early stages $(1 \& 2)$. CTC, circulating tumour cell.

stages as shown in Figure 6.

\section{Sensitivity and specificity for different assay methodology}

Reverse transcription polymerase chain reaction had the highest sensitivity for AFP with sensitivity of $97 \%$ whilst MS PCR had the highest specificity of $99 \%$. Chemiluminescence and Elisa had moderate sensitivity $70 \%, 67 \%$ and specificity $57 \%$ and $75 \%$ respectively. Most of the other CTC studies used RT- PCR, a total of 15 studies were identified. RT PCR shown to produce a high specificity above 0.6 but a moderate sensitivity.

\section{Positivity of early vs. late stages cancers in CTC detection}

AFP mRNA, cytokeratin-19 (Ck19), cluster differentiation (CD90), cfDNA and tissue factor pathway inhibitor-2 (TFPI2) all indicated CTC show a higher percentage of positivity in later stages in comparison to earlier stages. There was a difference of $6 \%, 44 \%, 27 \%, 5 \%$ and $13 \%$ respectively. However other CTC such as cfDNA show no difference. Interesting MT1G shows higher positivity in lower stages in comparison to higher stages.

\section{Sensitivity and specificity for the different cutoff values}

Han et al. (21) and Huang et al. (24) explored the effect of various cut off to the sensitivity and specificity of the diagnostic test. In the study of AFP, a higher cut off levels (400 ng/mL) was found to be more specific (99\%) to lower levels (20 ng/mL) (65\%), whilst being less sensitive (24\% to $58 \%$ ). Similar results were seen in TRG5+ AFP (specificity of $90 \%$ to $65 \%$ and sensitivity of $65 \%$ to $81 \%$ ) and $P 16$ INK 4 A (specificity of $98 \%$ to $81 \%$ and sensitivity of $27 \%$ to $74 \%$ ) (Figure 4 ).

\section{Youden index}

Youden index is summary statistic of the roc curve used in the interpretation and evaluation of biomarkers. A value of zero indicates the diagnostic test gives a positive result for those with or without the disease and a value of 1 indicates no false positive or false negative. An acceptably benchmark is 0.50 (50). In this study, the highest index of 0.87 was produced by Bahnassy et al. (15) who studied AFP. In contrast, Iizuka et al. (28) analyzed SPINT2 that was found to have the lowest index of 0.01. The average index amongst all CTC was 0.46 with a mode and median of 0.5 . In comparing, 5 most common CTC that is AFP, 249serP54, P16, RASSF1A and cfDNA, CfDNA had the highest average index of 0.53 followed by RASSF1A (0.41), P16 (0.40). Both AFP and. 249 serP54 had the worst overall index of 0.28 (Table 1).

\section{Discussion}

Blood based biomarkers could have promising value in early 
diagnosis of HCC and therefore allow prompt treatment (51). It could be used a less-invasive alternative to current approach in diagnosis. However despite the range of CTC currently under investigation (52), there is variation in the reported diagnostic accuracy and the lack of standardized technical approach has contributed to the lack of consensus.

In a recent update in the Cochrane methods of screening and diagnostics tests, the current statistical model used in meta-analysis of diagnostic accuracy is SROC curves and the use of pooled sensitivity and specificity is considered an accurate method of reporting of such data (53).

The following criteria was used to evaluate the pooled sensitivity and specificity: high (0.6-1), moderate (0.4-0.59), low $(<0.4)(51)$. In this meta-analysis we found that AFP has the highest overall diagnostic performance. The most common CTCs currently studied (249ser P53, P16, cfDNA and RASSF1A) have low to average Youden Index 0.28 to 0.56. Interestingly whilst Bahnassy et al. (15) demonstrated the highest overall Youden index using AFP, the average index of AFP was 0.28. This may result from different cutoff used or assay method. Further studies are needed to better understand this.

From our available statistical analysis, the study demonstrated that liquid biopsies have a high sensitivity/ specificity however there is several limitations. This study has identified several heterogenous variables such as the follows: First demographic data (age, sex and race), sample size and etiology of HCC which was missing in the data. In addition, the underlying etiology of $\mathrm{HCC}$ was variable among and within studies.

There were inconsistencies in cut off values used for individual CTC's, therefore the sensitivity and specificity could have been over or underestimated as shown in Figure 4. Unsurprisingly, the higher the cutoff value the higher the specificity but lower sensitivity due to higher rates of false negatives. For future studies, a singles cutoff value should be determined for each CTC to reduce outcome bias.

In addition, assay methods for CTC detection have shown to produce different results for the same type of liquid biopsy. A different cutoff value and varied experimental set up may account for these findings, however from our results we can take into consideration that different CTC detection methods of the same CTC may potentially create bias. Currently the standard for CTC detection immunocytochemistry (ICC) and reverse transcriptase polymerase chain reaction (RT-PCR).

We were unable to identify complete data sets. True positive, false positive, True Negative or false negative of various articles were calculated using the available sensitivity and specificity.

Finally our study sample size per CTC was too small which limited our ability to complete a full SROC curve thus the analysis from the SROC curve provided in this study was descriptive.

Overall, there is potential in the use of CTC however the lack of a standardized procedure in the study of CTC contributes to the lack of consensus of its use.

Future research should include large scaled, standardized studies for the diagnostic accuracy of CTC. Only when such a challenge is met should it be translated these promising results to clinical practice.

\section{Conclusions}

The CTC markers have variable sensitivity and specificity for HCC. CD133 and CK19 could potentially be used to differentiate early versus late stages irrespective to the morphology. Further studies are required to establish it use as an isolated test for detection. Therefore, in the current clinical context, CTCs must be used with other clinical investigations.

\section{Acknowledgments}

Funding: None.

\section{Footnote}

Conflicts of Interest: All authors have completed the ICMJE uniform disclosure form (available at http://dx.doi. org/10.21037/tgh.2020.01.11). The authors have no conflicts of interest to declare.

Ethical Statement: The authors are accountable for all aspects of the work in ensuring that questions related to the accuracy or integrity of any part of the work are appropriately investigated and resolved.

Open Access Statement: This is an Open Access article distributed in accordance with the Creative Commons Attribution-NonCommercial-NoDerivs 4.0 International License (CC BY-NC-ND 4.0), which permits the noncommercial replication and distribution of the article with the strict proviso that no changes or edits are made and the original work is properly cited (including links to both the formal publication through the relevant DOI and the license). See: https://creativecommons.org/licenses/by-nc$\mathrm{nd} / 4.0 \%$. 


\section{References}

1. Bosch FX, Ribes J, Cléries R, et al. Epidemiology of hepatocellular carcinoma. Clin Liver Dis 2005;9:191-211.

2. El-Serag HB. Epidemiology of viral hepatitis and hepatocellular carcinoma. Gastroenterology 2012;142:1264-1273.e1.

3. Sánchez Antolín G, García Pajares F, Pérez E, et al. Milan Criteria Versus San Francisco Criteria in Hepatocellular Carcinoma: Our Center's Experience. Transplant Proc 2009;41:1012-3.

4. Liu C, Duan LG, Lu WS, et al. Prognosis evaluation in patients with hepatocellular carcinoma after hepatectomy: Comparison of BCLC, TNM and Hangzhou criteria staging systems. PLoS One 2014;9:e103228.

5. Yu NC, Chaudhari V, Raman SS, et al. CT and MRI Improve Detection of Hepatocellular Carcinoma, Compared With Ultrasound Alone, in Patients With Cirrhosis. Clin Gastroenterol Hepatol 2011;9:161-7.

6. Wang G, Zhu S, Li X. Comparison of values of CT and MRI imaging in the diagnosis of hepatocellular carcinoma and analysis of prognostic factors. Oncol Lett 2019;17:1184-8.

7. Vona G, Estepa L, Béroud C, et al. Impact of cytomorphological detection of circulating tumor cells in patients with liver cancer. Hepatology 2004;39:792-7.

8. Tokuhisa Y, Iizuka N, Sakaida I, et al. Circulating cellfree DNA as a predictive marker for distant metastasis of hepatitis $\mathrm{C}$ virus-related hepatocellular carcinoma. $\mathrm{Br} \mathrm{J}$ Cancer 2007;97:1399-403.

9. Welch V, Petticrew M, Tugwell P, et al. PRISMA-Equity 2012 Extension: Reporting Guidelines for Systematic Reviews with a Focus on Health Equity. PLoS Med 2012;9:e1001333.

10. da Costa Santos CM, de Mattos Pimenta CA, Nobre MR. The PICO strategy for the research question construction and evidence search. Rev Lat Am Enfermagem 2007;15:508-11.

11. Ouzzani M, Hammady H, Fedorowicz Z, et al. Rayyan-a web and mobile app for systematic reviews. Syst Rev 2016;5:210.

12. Zaugg H, West RE, Tateishi I, et al. Mendeley: Creating communities of scholarly inquiry through research collaboration. Tech Trends 2011;55:32-6.

13. Whiting PF, Weswood ME, Rutjes AW, et al. Evaluation of QUADAS, a tool for the quality assessment of diagnostic accuracy studies. BMC Med Res Methodol 2006;6:9.

14. Aselmann H, Wolfes H, Rohde F, et al. Quantification of alpha 1-fetoprotein mRNA in peripheral blood and bone marrow: a tool for perioperative evaluation of patients with hepatocellular carcinoma. Langenbecks Arch Surg 2001;386:118-23.

15. Bahnassy AA, Zekri A-RN, El-Bastawisy A, et al. Circulating tumor and cancer stem cells in hepatitis $\mathrm{C}$ virus-associated liver disease. World J Gastroenterol 2014;20:18240-8.

16. Chang H, Yi B, Li L, et al. Methylation of tumor associated genes in tissue and plasma samples from liver disease patients. Exp Mol Pathol 2008;85:96-100.

17. Chen H, Sun LY, Zheng HQ, et al. Total serum DNA and DNA integrity: Diagnostic value in patients with hepatitis $B$ virus-related hepatocellular carcinoma. Pathology 2012;44:318-24.

18. Chen K, Zhang H, Zhang LN, et al. Value of circulating cell-free DNA in diagnosis of hepatocelluar carcinoma. World J Gastroenterol 2013;19:3143-9.

19. Chu HJ, Heo J, Seo SB, et al. Detection of Aberrant p16INK4A Methylation in Sera of Patients with Liver Cirrhosis and Hepatocellular Carcinoma. J Korean Med Sci 2004;19:83-6.

20. Fu Y, Xu X, Huang D, et al. Plasma Heat Shock Protein 90alpha as a Biomarker for the Diagnosis of Liver Cancer: An Official, Large-scale, and Multicenter Clinical Trial. EBioMedicine 2017;24:56-63.

21. Han LY, Fan YC, Mu NN, et al. Aberrant DNA methylation of G-protein-coupled bile acid receptor gpbar1 (TGR5) is a potential biomarker for hepatitis B virus associated hepatocellular carcinoma. Int J Med Sci 2014;11:164-71.

22. Hosny G, Farahat N, Tayel H, et al. Ser-249 TP53 and CTNNB1 mutations in circulating free DNA of Egyptian patients with hepatocellular carcinoma versus chronic liver diseases. Cancer Lett 2008;264:201-8.

23. Huang XH, Sun LH, Lu DD, et al. Codon 249 mutation in exon 7 of $\mathrm{p} 53$ gene in plasma DNA: maybe a new early diagnostic marker of hepatocellular carcinoma in Qidong risk area, China. World J Gastroenterol 2003;9:692-5.

24. Huang G, Krocker JD, Genetic M, et al. Evaluation of INK4A promoter methylation using pyrosequencing and circulating cell-free DNA from patients with hepatocellular carcinoma. Clinical Chemistry and Laboratory Medicine (CCLM) 2014;52:899-909.

25. Huang Z, Hua D, Hu Y, et al. Quantitation of plasma circulating DNA using quantitative PCR for the detection of hepatocellular carcinoma. Pathol Oncol Res 2012;18:271-6.

26. Igetei R, Otegbayo JA, Ndububa DA, et al. Detection 
of p53 codon 249 mutation in Nigerian patients with hepatocellular carcinoma using a novel evaluation of cellfree DNA. Ann Hepatol 2008;7:339-44.

27. Iizuka N, Sakaida I, Moribe T, et al. Elevated levels of circulating cell-free DNA in the blood of patients with hepatitis $\mathrm{C}$ virus-associated hepatocellular carcinoma. Anticancer Res 2006;26:4713-9.

28. Iizuka N, Oka M, Sakaida I, et al. Efficient detection of hepatocellular carcinoma by a hybrid blood test of epigenetic and classical protein markers. Clin Chim Acta 2011;412:152-8.

29. Jeng KS, Sheen IS, Tsai YC. Does the presence of circulating hepatocellular carcinoma cells indicate a risk of recurrence after resection? Am J Gastroenterol 2004;99:1503-9.

30. Ji XF, Fan YC, Gao S, et al. MT1M and MT1G promoter methylation as biomarkers for hepatocellular carcinoma. World J Gastroenterol 2014;20:4723-9.

31. Jiang P, Chan CWM, Chan KCA, et al. Lengthening and shortening of plasma DNA in hepatocellular carcinoma patients. Proc Natl Acad Sci U S A 2015;112:E1317-25.

32. Julich-Haertel H, Urban SK, Krawczyk M, et al. Cancerassociated circulating large extracellular vesicles in cholangiocarcinoma and hepatocellular carcinoma. J Hepatol 2017;67:282-92.

33. Kirk GD, Mendy M, Goedert JJ, et al. Ser-249 p53 mutations in plasma DNA of patients with hepatocellular carcinoma from The Gambia. J Natl Cancer Inst 2000;92:148-53.

34. Kuo CC, Lin CY, Shih YL, et al. Frequent methylation of HOXA9 gene in tumor tissues and plasma samples from human hepatocellular carcinomas. Clin Chem Lab Med 2014;52:1235-45.

35. Mohamed NA, Swify EM, Amin NF, et al. Is serum level of methylated RASSF1A valuable in diagnosing hepatocellular carcinoma in patients with chronic viral hepatitis C? Arab J Gastroenterol 2012;13:111-5.

36. Müller C, Petermann D, Pfeffel F, et al. Lack of specificity of albumin-mRNA-positive cells as a marker of circulating hepatoma cells. Hepatology 1997;25:896-9.

37. Piciocchi M, Cardin R, Vitale A, et al. Circulating free DNA in the progression of liver damage to hepatocellular carcinoma. Hepatol Int 2013;7:1050-7.

38. Ren N, Qin LX, Tu H, et al. The prognostic value of circulating plasma DNA level and its allelic imbalance on chromosome $8 \mathrm{p}$ in patients with hepatocellular carcinoma. J Cancer Res Clin Oncol 2006;132:399-407.

39. Schulze K, Gasch C, Staufer K, et al. Presence of EpCAM- positive circulating tumor cells as biomarker for systemic disease strongly correlates to survival in patients with hepatocellular carcinoma. Int J Cancer 2013;133:2165-71.

40. Sun FK, Fan YC, Zhao J, et al. Detection of TFPI2 methylation in the serum of hepatocellular carcinoma patients. Dig Dis Sci 2013;58:1010-5.

41. Tan SH, Ida H, Lau QC, et al. Detection of promoter hypermethylation in serum samples of cancer patients by methylation-specific polymerase chain reaction for tumour suppressor genes including RUNX3. Oncol Rep 2007;18:1225-30.

42. Witzigmann H, Geissler F, Benedix F, et al. Prospective evaluation of circulating hepatocytes by alpha-fetoprotein messenger RNA in patients with hepatocellular carcinoma. Surgery 2002;131:34-43.

43. Wong IHN, Zhang J, Lai PBS, et al. Quantitative analysis of tumor-derived methylated p16INK4a sequences in plasma, serum, and blood cells of hepatocellular carcinoma patients. Clin Cancer Res 2003;9:1047-52.

44. Briefs T, Pathology C, Pathology C. Relationship of p16 methylation status and serum alpha-fetoprotein concentration in hepatocellular carcinoma patients. 1998:1420-2.

45. Yang SZ, Dong JH, Li K, et al. Detection of AFPmRNA and melanoma antigen gene-1mRNA as markers of disseminated hepatocellular carcinoma cells in blood. Hepatobiliary Pancreat Dis Int 2005;4:227-33.

46. Okajima W, Komatsu S, Ichikawa D, et al. Liquid biopsy in patients with hepatocellular carcinoma: Circulating tumor cells and cell-free nucleic acids. World J Gastroenterol 2017;23:5650-68.

47. Yeo W, Wong N, Wong WL, et al. High frequency of promoter hypermethylation of RASSF1A in tumor and plasma of patients with hepatocellular carcinoma. Liver Int 2005;25:266-72.

48. Zhang P, Wen X, Gu F, et al. Methylation profiling of serum DNA from hepatocellular carcinoma patients using an Infinium Human Methylation 450 BeadChip. Hepatol Int 2013;7:893-900.

49. Zhang YJ, Wu HC, Shen J, et al. Predicting hepatocellular carcinoma by detection of aberrant promoter methylation in serum DNA. Clin Cancer Res 2007;13:2378-84.

50. Ruopp MD, Perkins NJ, Whitcomb BW, et al. Youden Index and optimal cut-point estimated from observations affected by a lower limit of detection. Biom J 2008;50:419-30.

51. Behne T, Copur MS. Biomarkers for Hepatocellular Carcinoma. Int J Hepatol 2012;2012:859076. 
52. Quandt D, Dieter Zucht H, Amann A, et al. Implementing liquid biopsies into clinical decision making for cancer immunotherapy. Oncotarget 2017;8:48507-20.

doi: $10.21037 / \operatorname{tgh} .2020 .01 .11$

Cite this article as: Tan P, Grundy L, Makary P, Eng KH, Ramsay G, Bekheit M. The value of liquid biopsy in the diagnosis and staging of hepatocellular carcinoma: a systematic review. Transl Gastroenterol Hepatol 2021;6:54.
53. Leeflang MM, Deeks JJ, Takwoingi Y, et al. Cochrane diagnostic test accuracy reviews. Syst Rev 2013;2:82. 